\title{
Estrogen Status and Heredity Are Major Determinants of Premenopausal Bone Mass
}

Reina Armamento-Villareal, Dennis T. Villareal, Louis V. Avioli, and Roberto Civitelli Division of Endocrinology and Bone and Mineral Diseases, the Jewish Hospital of St. Louis at Washington University Medical Center, St. Louis, Missouri 63110

\section{Abstract}

To analyze their relative effects on premenopausal bone mass, we have studied the impact of lifelong estrogen exposure, assessed by an estrogen score ( $E S$; computed on age at menarche, average length of menstrual cycles since menarche, and use of birth control pills), heredity, and some environmental factors on vertebral bone density (VBD), of 63 premenopausal women (age, 19-40 yr). Compared with women with normal bone density $(Z$ score $>-1)$, subjects with low VBD $(Z$ score $<-1)$ had significantly lower $\mathrm{ES}(15.1 \pm 3.9$ vs. $18.7 \pm 2.4, P=0.001)$, higher age at menarche (13.8 \pm 1.7 vs. $12.6 \pm 1.4 \mathrm{yr}, P=0.005)$, and lower serum estradiol $(46.9 \pm 37$ vs. $86.6 \pm 57 \mathrm{pg} / \mathrm{ml}, P$ $=0.023)$ and estrone levels $(107.4 \pm 60$ vs. $178.8 \pm 9.0 \mathrm{pg} / \mathrm{ml}, P$ $=0.05)$. Likewise, women in the lowest quartile for VBD had significantly lower ES $(15.3 \pm 4.5$ vs. $18.1 \pm 2.7, P=0.006)$ and higher age at menarche $(13.9 \pm 1.9$ vs. $12.8 \pm .4, P=0.02)$ than those in the upper three quartiles. $A$ higher proportion of subjects with irregular menses ( 52 vs. $23 \%, P=0.03)$ and a positive family history of osteoporosis ( 86 vs. $61 \%, P=0.04$ ) was found in the low VBD group compared with subjects with normal VBD. VBD correlated positively with ES $(r=0.44, P$ $=<0.001)$ and negatively with age at menarche $(r=-0.30, P$ $=0.03$ ) by simple linear regression, whereas no correlation was found between VBD and age, body mass index, parity, lactation, physical activity, sunlight exposure, and dietary calcium and vitamin D intakes. The correlation between VBD and ES improved after correcting for the effect of all the other variables by partial correlation analysis (Pearson partial $r=0.57, P$ $=<0.01$ ), which also disclosed a significant contribution of dietary calcium to VBD. However, ES was the only significant independent determinant of VBD, by stepwise multiple regression analysis $\left(R^{2}=0.24\right)$. Therefore, premenopausal estrogen exposure, and possibly genetic predisposition, rather than environmental factors, are the major determinants for the development of peak bone mass before menopause. (J. Clin. Invest. 1992. 90:2464-2471.) Key words: bone density • osteoporosis • menstrual cycle $\bullet$ menarche $\bullet$ menopause

Address correspondence to Reina Armamento-Villareal, M.D., Division of Endocrinology and Bone Metabolism, The Jewish Hospital of St. Louis, 216 South Kingshighway, St. Louis, MO 63110. Address reprint requests to Roberto Civitelli, M.D., at the same address. 1992.

Received for publication 4 April 1992 and in revised form 8 June

J. Clin. Invest.

(c) The American Society for Clinical Investigation, Inc. 0021-9738/92/12/2464/08 \$2.00

Volume 90, December 1992, 2464-2471

\section{Introduction}

Postmenopausal bone mineral density is dependent on both the amount of bone mass accumulated during infancy and adolescence and the rate of bone loss that occurs with menopause and aging (1). Since pharmacological interventions currently in vogue for the treatment of established osteoporotic syndromes can only maintain bone mass, or at best achieve modest transient increases (2-4), prevention of bone loss with early intervention currently appears to be the best strategy for the management of osteoporosis. In fact, osteoporosis prevention programs could be directed not only to reduce the rapid bone loss that follows menopause but also to potentiate the accumulation of bone mass during infancy through early adulthood. Ideally, the achievement of an adequate peak bone mass at skeletal maturity should constitute the best protection against potential future bone loss. Hence, the identification of factors that influence the development of optimal bone density are of utmost importance, since this information may aid researchers and clinicians to devise the most appropriate strategies for maximizing bone mass in the developmental period.

Both genetic and environmental factors have been invoked as determinants of peak bone mass $(5-8,11,14,15,32,37)$. A relevant role of heredity has been recently underscored by observations of reduced bone densities in daughters of women with osteoporosis compared with those of normal mothers (6) and from twin studies showing greater concordance in bone densities between monozygotic than dizygotic twins $(7,8)$. Since this concordance decreases as the twin pairs age, it appears that factors related to the environment play an increasingly important role as aging ensues. Postmenopausal bone loss is clearly related to estrogen deficiency (9) and can be prevented by estrogen replacement (3); therefore, one can speculate that in females estrogens may play a functional role in the achievement and maintenance of bone mass during the fertile period. Since information on the role of estrogens in premenopausal women is still scanty and inconclusive (10-12), this study was designed to evaluate the relative contribution of estrogen status, parity, lactation, family history of osteoporosis, as well as dietary and other environmental factors to bone mass in premenopausal women. The results indicate that estrogen status is the most important determinant of premenopausal bone mass in Caucasian females and underscore the importance of genetic over environmental factors for the acquisition and maintenance of an adequate bone mass before the menopause.

\section{Methods}

Experimental subjects. Cross-sectional data of 84 premenopausal Caucasian women referred to the Bone Health Program of The Jewish Hospital of St. Louis, Washington University Medical Center during the years 1986-1990 for screening of osteoporosis were reviewed. Sub- 
jects affected by disorders of mineral metabolism or by conditions known to cause secondary osteoporosis, such as hyperthyroidism, cortisol excess, malabsorption syndromes, and malignancies, were excluded. Likewise, history of alcohol abuse, smoking, and intake of drugs known to affect calcium metabolism, including corticosteroids, thyroid hormones, anticonvulsants, diuretics, as well as bisphosphonates, fluorides, and calcitonin were considered exclusion criteria. 21 of the subjects screened were thus excluded from the analysis; the remaining 63 women constituted the study population.

Family history. Information on family history of osteoporosis was obtained during medical interview. History was considered to be positive if any one of the following criteria was met in a first-degree relative on the mother's side: evidence of osteoporosis by $\mathrm{x}$-rays or, when available, bone densitometry; presence of radiologically confirmed nontraumatic fractures; presence of severe kyphosis; absence of disorders of bone metabolism; and intake of substances known to affect skeletal homeostasis, according to the same inclusion/exclusion criteria detailed for the study population.

Anthropometric data. Standing height and weight were recorded. Physical build was expressed as body mass index (BMI), ${ }^{1}$ calculated as the weight (in kilograms) divided by the square of the height (in meters).

Physical activity. Information about each women's physical activity was obtained by a questionnaire devised to reflect the average lifelong lifestyle of each subject. Physical activity was categorized as low moderate, and high and was scored as follows: low activity (grade 1), mostly sedentary lifestyle, that is, being on feet $<50 \%$ of the time without performing a regular set of exercises; moderate activity (grade 2 ), being on feet $50-75 \%$ of the time or performing a regular set of exercises, such as jogging, walking, biking, and aerobics, for $\geq 30 \mathrm{~min} /$ $\mathrm{d}$ and $\geq 2 \mathrm{~d} / \mathrm{wk}$; high activity (grade 3 ), being on feet $>75 \%$ of the time or engaging in regular heavy work, sports, or exercises for $>1 \mathrm{~h} / \mathrm{d}$ and $>4 \mathrm{~d} /$ wk.

Estrogen exposure. To quantitate a woman's lifelong exposure to estrogen, a scoring system was developed by modifying the method described by Dhuper et al. (13). This estrogen score (ES) is based on the three main physiological and environmental factors that affect the estrogen status of a fertile woman: duration of the fertile period (expressed as age at menarche), length and frequency of menstrual cycles, and use of birth control medications. Weighted scores were assigned for each factor as shown in Table I. Accordingly, each individual could score between 2 and 24 points. A normal menstrual cycle (eumenorrhea) was defined as the occurrence of 9-13 menses/yr. Between three and eight menses/yr was considered oligomenorrhea and less than three menses/yr was considered amenorrhea. Accordingly, a subject was classified as having had menstrual irregularities when at least one episode of either oligomenorrhea or amenorrhea was reported during the fertile period. However, for the ES, periods or transient oligomenhorrhea and amenorrhea were scored according to the total duration for each subject's fertile period (Table I). Age at menarche and menstrual cycles were considered the major determinants of a woman's overall estrogen exposure and, thus, they were given the greatest weight. Since available data are inconclusive as to whether oral contraceptives have a significant impact on bone density (14-17) the use of birth control pills was given a lower weight in our scoring system, although scores were adjusted for the duration of use.

Dietary intake. Assessment of dietary habits was performed by a registered dietitian using the cross-check method (18). The subjects were asked to indicate weekly frequency of consumption of certain food products. Records of a typical daily intake were then used to cross-check the food frequency data. They were entered in a database and elaborated by a computer program (IPC data diet, San Jose, CA), which calculates the relative content of each dietary element from a

1. Abbreviations used in this paper: BMI, body mass index; E1, estrone; E2, estradiol; ES, estrogen score; VBD, vertebral bone density.
Table I. Estrogen Score

\begin{tabular}{|c|c|}
\hline Parameter & Score \\
\hline \multicolumn{2}{|l|}{ Age at menarche $(\mathrm{yr})$} \\
\hline$\leq 10$ & 10 \\
\hline 11 & 9 \\
\hline 12 & 8 \\
\hline 13 & 7 \\
\hline 14 & 6 \\
\hline 15 & 5 \\
\hline 16 & 4 \\
\hline 17 & 3 \\
\hline 18 & 2 \\
\hline 19 & 1 \\
\hline$\geq 20$ & 0 \\
\hline \multicolumn{2}{|l|}{ Menstrual cycle } \\
\hline Eumenorrheic & 10 \\
\hline \multicolumn{2}{|c|}{ Oligomenorrheic (total mo) } \\
\hline$<6$ & 8 \\
\hline $6-12$ & 6 \\
\hline$>12$ & 4 \\
\hline \multicolumn{2}{|c|}{ Amenorrheic (total mo) } \\
\hline$<6$ & 6 \\
\hline $6-12$ & 4 \\
\hline$>12$ & 2 \\
\hline \multicolumn{2}{|c|}{ Use of birth control pills (yr) } \\
\hline$<1$ & 1 \\
\hline $1-3$ & 2 \\
\hline$>3-5$ & 3 \\
\hline$>5$ & 4 \\
\hline
\end{tabular}

typical daily diet. The average daily intake of calcium and vitamin D were recorded.

Sunlight exposure. Information on sunlight exposure was obtained through a questionnaire and expressed as outdoor score using a method described previously (18). Briefly, the subjects were asked how much time they spent outdoors in direct sunlight from 0900-1600, during $1 \mathrm{~d}$ in the different seasons of the year. Scores were assigned as follows: not at all in any season, 0 ; $<30 \mathrm{~min}$ : winter, 0 ; spring, 2; fall, 2; summer, 4; $>30$ min: winter, 1 ; spring, 3 ; fall, 3 ; summer, 5 . The sum of the points obtained for the four seasons $(0-12)$ constituted the outdoor score.

Bone densitometry. Vertebral bone density (VBD) was measured by quantitative computed tomography of the vertebral bodies of $\mathrm{T} 12$ to L3, using a scanner (GE 9800) $(19,20)$. The coefficient of variation is $4.5 \%$ in our center (20). VBD values were expressed either in milligrams per cubic centimeter, or as $Z$ scores, computed based on the data from 538 normal nonblack women (19) aged 20-80 yr, to indicate the deviation from the expected average values for sex- and age-matched normals.

Biochemical tests. Serum alkaline phosphatase and calcium, and urine calcium and creatinine tests, were performed using standard autoanalyzer techniques. Serum $25(\mathrm{OH}) \mathrm{D}$ was measured by a competitive protein-binding radioassay (21). Serum estrone (E1) and estradiol (E2) were measured using radioimmunoassay techniques (SmithKline Beecham Laboratories, Van Nuys, CA). Since serum samples for E1 and E2 were drawn at the time each subject presented for evaluation, E1 and E2 values are not related to a specific phase of the menstrual cycle.

Statistical analysis. Statistical analysis was performed using Statgraphics (version 5.0; STSC Inc., Rockville, MD). Data were managed using Lotus 1-2-3 (version 2.0; Lotus Development Corp., Cambridge, MA). Results are expressed as mean \pm standard deviation. The interactions between age, BMI, ES, E1, E2, age of menarche, number of preg- 
Table II. Clinical and Biochemical Characteristics of Patients Stratified by Age

\begin{tabular}{|c|c|c|c|c|c|}
\hline & $\begin{array}{c}\leq 25 \\
(n=4)\end{array}$ & $\begin{array}{c}26-30 \\
(n=7)\end{array}$ & $\begin{array}{c}31-35 \\
(n=18)\end{array}$ & $\begin{array}{c}36-40 \\
(n=34)\end{array}$ & $P^{*}$ \\
\hline $\operatorname{VBD}\left(\mathrm{mg} / \mathrm{cm}^{3}\right)$ & $164.3 \pm 30$ & $145.0 \pm 20$ & $151.1 \pm 2.8$ & $154.3 \pm 29$ & NS \\
\hline BMI & $19.3 \pm 1.2$ & $19.5 \pm 1.8$ & $21.7 \pm 3.1$ & $21.9 \pm 3.4$ & NS \\
\hline Family history (\% positive) & 33 & 66 & 87 & 66 & NS \\
\hline Activity score & $1.3 \pm 0.5^{\ddagger}$ & $2.0 \pm 0.63$ & $2.1 \pm 0.5$ & $2.0 \pm 0.5$ & 0.026 \\
\hline Outdoor score & $11.3 \pm 1.0$ & $10.8 \pm 1.3$ & $11.3 \pm 0.9$ & $10.5 \pm 1.6$ & NS \\
\hline Dietary Ca (mg/d) & $683 \pm 183$ & $1,123 \pm 557$ & $1,004 \pm 494$ & $947 \pm 469$ & NS \\
\hline Dietary vitamin D (IU/d) & $103 \pm 110$ & $188 \pm 133$ & $167 \pm 129$ & $212 \pm 164$ & NS \\
\hline Caloric intake (kcal/d) & $1904 \pm 372$ & $1,803 \pm 369$ & $1,714 \pm 457$ & $1,660 \pm 248$ & NS \\
\hline Serum $25(\mathrm{OH}) \mathrm{D}(\mathrm{ng} / \mathrm{ml})$ & $35.0 \pm 35$ & $24.8 \pm 10$ & $28.2 \pm 9$ & $27.5 \pm 9$ & NS \\
\hline Alkaline phosphatase (IU/liter) & $63.3 \pm 21$ & $41.0 \pm 12$ & $56.0 \pm 19$ & $48.3 \pm 13$ & NS \\
\hline Urine $\mathrm{Ca}(\mathrm{mg} / 24 \mathrm{~h})$ & $94 \pm 42$ & $170 \pm 113$ & $131 \pm 79$ & $139 \pm 66$ & NS \\
\hline ES & $17.7 \pm 1.5$ & $17.5 \pm 4.5$ & $17.4 \pm 4.2$ & $17.4 \pm 3.0$ & NS \\
\hline Age at menarche (yr) & $12.7 \pm 1.2$ & $12.2 \pm 1.3$ & $13.1 \pm 1.9$ & $13.2 \pm 1.6$ & NS \\
\hline Menstrual irregularities (\% positive) & 25 & 17 & 39 & 26 & NS \\
\hline $\mathrm{BCP}$ (yr of use) & $1.4 \pm 2.4$ & $3.5 \pm 3.5$ & $4.4 \pm 3.7$ & $3.9 \pm 3.5$ & NS \\
\hline Serum estradiol (pg/ml) & NA & $63.0 \pm 30$ & $55.7 \pm 54$ & $90.2 \pm 61$ & NS \\
\hline Serum estrone $(\mathrm{pg} / \mathrm{ml})$ & NA & $125.5 \pm 55$ & $146.8 \pm 83$ & $175.0 \pm 89$ & NS \\
\hline Number of pregnancies & $1.0 \pm 2.0$ & $0.5 \pm 0.8$ & $1.5 \pm 1.5$ & $1.4 \pm 1.3$ & NS \\
\hline Lactation (yr) & $0.8 \pm 1.5$ & $1.5 \pm 3.8$ & $7.7 \pm 11.6^{\ddagger}$ & $1.7 \pm 3.5$ & 0.032 \\
\hline
\end{tabular}

NA, not available. * One-way analysis of variance. ${ }^{\ddagger}$ Different than all the other groups (Tukey’s multiple-range test).

nancies, duration of lactation, dietary factors, biochemical data, and VBD were analyzed by analysis of variance and, when appropriate, Tukey's LSD multiple-range test, as described in Results and legends to the Tables. In some cases, group means were compared using the twotailed non-paired Student's $t$ test. Binary variables and frequency distributions were analyzed using Chi-square for contingency tables. Simple and Pearson partial correlation analyses were employed to study the relationships between ES, E1, E2, and age of menarche with VBD. Stepwise multiple regression analysis was used to assess the independent contribution of each variable to bone density.

\section{Results}

Initially, the effect of age on bone density and the other variables was studied. To this end, the study population was divided into four 5-yr groups (Table II) and the data were analyzed by one-way analysis of variance. No significant differences were observed across the age groups for all parameters, with the exception of physical activity score and lactation. Multiple-range test disclosed a significantly lower level of activity in the youngest women and a higher number of years of lactation in the 31-35-yr-old subjects. However, the former finding may only reflect a sample bias because of the limited number of subjects included in the first group. Interestingly, the average bone mineral density was not different across the four age groups. Accordingly, no correlation was found between age and VBD by linear regression $(r<0.10)$ (Fig. 1). Although this result would rule out significant age-related changes in bone mass in this cohort, the subsequent analyses were still performed on data normalized to the age- and sex-adjusted expected average VBD.

To identify potential factors that can contribute to premenopausal bone mass, the population was stratified according to VBD $Z$ scores using two approaches. First, two groups were generated using a cut-off value of 1 SD below the age-adjusted average bone density. By this method the population was divided approximately at the lower third. Thus, the group with normal bone density $(Z$ score $>-1)$ was about twice as large as the low bone density group ( $Z$ score $<-1$; Table III). No significant differences were observed in age, body mass, physical activity, sunlight exposure, nutritional habits, and biochemical indices. However, there was a significantly higher proportion of subjects with positive family history of osteoporosis in the low bone density group. Furthermore, the latter group had an average lower estrogen score, higher age at menarche, higher incidence of irregular menses, and lower random E2 and E1 levels (Table IV). On the other hand, years of use of birth control pills, parity, and average duration of lactation per woman were not different between the two groups.

As a second approach, the study population was stratified in quartiles based on VBD $Z$ scores. This method produces four groups of equal numbers. Results closely similar to those

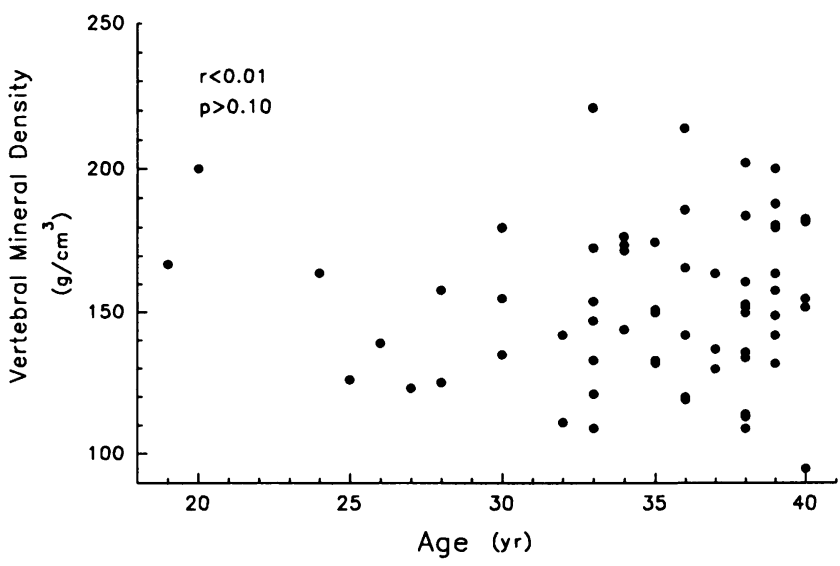

Figure 1. Age-related distribution of vertebral bone density (T12-L3) in 63 premenopausal Caucasian women. 
Table III. Clinical and Biochemical Characteristics of Patients Stratified by $B M D$

\begin{tabular}{lccc}
\hline \multicolumn{1}{c}{ Parameter } & $\begin{array}{c}\text { BMD }>-1 \text { SD } \\
(n=40)\end{array}$ & $\begin{array}{c}\text { BMD }<-1 \text { SD } \\
(n=23)\end{array}$ & $P^{*}$ \\
\hline Age (yr) & $34.9 \pm 5.1$ & $34.3 \pm 4.5$ & NS \\
BMI & $21.3 \pm 2.5$ & $21.3 \pm 4.0$ & NS \\
Family history & & & \\
$\quad$ \% of total) & 61.5 & 86.9 & $0.014^{\ddagger}$ \\
Activity score & $2.0 \pm 0.5$ & $1.9 \pm 0.5$ & NS \\
Outdoor score & $10.8 \pm 1.5$ & $10.9 \pm 1.2$ & NS \\
Dietary Ca (mg/d) & $972.0 \pm 438.9$ & $961.9 \pm 531.2$ & NS \\
Dietary vitamin D & & & \\
$\quad(I U / d)$ & $168.2 \pm 115.9$ & $217.9 \pm 178.3$ & NS \\
Caloric intake & & & \\
$\quad$ kcal/d) & $1,694.2 \pm 385.9$ & $1,733.6 \pm 277.7$ & NS \\
Serum 25(OH)D & & & \\
$\quad(\mathrm{ng} / \mathrm{ml})$ & $27.8 \pm 10.2$ & $28.4 \pm 8.1$ & NS \\
Alkaline phosphatase & & & \\
$\quad$ (IU/liter) & $49.6 \pm 16.1$ & $52.8 \pm 15.6$ & NS \\
Serum Ca (mg/dl) & $9.2 \pm 0.5$ & $9.1 \pm 0.3$ & NS \\
Urinary Ca (mg/24 h) & $130.0 \pm 73.5$ & $149.9 \pm 78.7$ & NS \\
& & & \\
\hline
\end{tabular}

* Student's $t$ test for unpaired samples.

${ }^{\ddagger} \chi^{2}$ for a $2 \times 2$ contingency table.

just described were obtained by analysis of variance of each variable. Accordingly, a positive family history of osteoporosis was more common in the lower quartiles and less common in the higher quartiles (Table V). In particular, the incidence of familiarity was significantly higher in subjects in the lowest quartile than in the pooled quartiles $2-4$, whereas no difference was observed for any of the anthropometric, lifestyle, dietary, and biochemical parameters. Also, subjects in the lowest quartile had a significantly lower ES compared with those in both the highest quartile and the upper three quartiles combined (Table VI). Subjects in the lowest quartile also had a higher age at menarche than the average of all other subjects. Although the average serum estrogen levels tended to increase with increasing quartiles, the changes did not reach statistical significance, except for serum E1, which was higher in quartile 4 than in any other groups.

To further investigate the factors contributing to premenopausal bone mass, data on continuous variables were analyzed by simple and multiple-regression analyses. Considering the whole population, we found a significant positive correlation between VBD and estrogen score $(r=0.44, P<0.001$; Fig. 2$)$ and an inverse correlation between VBD and age at menarche ( $r=-0.30, P=0.03$; Fig. 3 ) by simple linear regression. Signifcant positive correlations were also observed for serum E2 ( $r$ $=0.50, P=0.02)$ and E1 $(r=0.48, P=0.03)$, although data from only 21 patients were available. On the other hand, no relationship was found between VBD and all the other parameters studied.

Data on a subset of 39 subjects, whose values on the most relevant parameters studied were complete, were analyzed further. The strong correlation between ES and VBD was confirmed in this subgroup by both simple regression and partial correlation analyses, which corrects for the interaction of any other variable included in the analysis (Table VII). Partial correlation also disclosed a significant contribution of dietary calcium and a surprisingly negative relationship between the dietary intake of vitamin $\mathrm{D}$ and bone density. Of the other variables, only the number of pregnancies and sunlight exposure gave sizable positive and negative partial $r$ values, respectively, but without reaching statistical significance.

Data from this subset of 39 women were also included in a multiple-regression model for stepwise selection of the variables contributing to VBD. As shown in Table VIII, the final model included only ES, which accounted for $24 \%$ of VBD variability. Forcing the next variable (dietary calcium) with the highest $F$ into the model only increased $R^{2}$ by $4 \%$; and including dietary vitamin $D$ added a further mere $4 \%$ to VBD variability. However, the high standard error of estimates and mean absolute error indicate that the final model, including only ES, is not a good predictor of bone mass. Nevertheless, these analyses confirm that estrogen status alone is the strongest independent determinant of premenopausal bone density in this group of Caucasian females.

\section{Discussion}

The present results support the hypothesis that estrogen exposure is the main factor determining bone mass in premenopausal women. Our data also suggest that genetic potential has a relatively higher weight than environmental factors in the development of premenopausal bone mass.

Other reports have also underscored the role of hormonal homeostasis in the acquisition and preservation of bone mass (22-24). Cann et al. (22) found that menstrual history is the best predictor of trabecular bone density in premenopausal women and that primary amenorrheic women have reduced bone densities compared with women with secondary amenorrhea (23), indicating that even transient or interrupted exposure to physiological levels of circulating estrogen is certainly better than total lack of sexual hormones. Along this line, Drinkwater et al. (24) recently observed lower bone densities in athletes with oligomenorrhea or amenorrhea as compared with eumenorrheic subjects, with the amenorrheic athletes having the most severe reduction in bone density. In our study, menstrual irregularities were found to be more frequent in women with lower bone mass, suggesting that periods of oligo-

Table IV. Estrogen Status of Patients Stratified by BMD

\begin{tabular}{lccl}
\hline \multicolumn{1}{c}{ Parameter } & BMD $>-1$ SD & BMD $<-1$ SD & $P^{*}$ \\
\hline ES & $18.7 \pm 2.4$ & $15.3 \pm 3.9$ & 0.0001 \\
Age at menarche $(\mathrm{yr})$ & $12.6 \pm 1.4$ & $13.8 \pm 1.7$ & 0.005 \\
Menstrual irregularities (\%) & 23.7 & 52.3 & $0.015^{\ddagger}$ \\
BCP (yr of use) & $3.9 \pm 3.3$ & $3.6 \pm 3.9$ & $\mathrm{NS}$ \\
Serum estradiol $(\mathrm{pg} / \mathrm{ml})$ & $86.6 \pm 57.1$ & $46.9 \pm 37$ & 0.023 \\
Serum estrone $(\mathrm{pg} / \mathrm{ml})$ & $178.8 \pm 9.0$ & $107.4 \pm 60.0$ & 0.05 \\
Number of pregnancies & $1.3 \pm 1.4$ & $1.3 \pm 1.1$ & $\mathrm{NS}$ \\
Lactation $(\mathrm{yr})$ & $3.8 \pm 7.7$ & $2.9 \pm 7.1$ & $\mathrm{NS}$ \\
& & & \\
\hline
\end{tabular}

* Student's $t$ test for unpaired samples.

${ }^{\ddagger} \chi^{2}$ for a $2 \times 2$ contingency table. 
Table V. Clinical and Biochemical Characteristics of Patients Stratified by Quartiles

\begin{tabular}{|c|c|c|c|c|c|}
\hline & $\begin{array}{l}\text { Quartile 1 } \\
(n=16)\end{array}$ & $\begin{array}{c}\text { Quartile 2 } \\
(n=16)\end{array}$ & $\begin{array}{c}\text { Quartile } 3 \\
(n=16)\end{array}$ & $\begin{array}{c}\text { Quartile 4 } \\
(n=16)\end{array}$ & $\begin{array}{l}\text { Quartiles 2-4 } \\
\quad(n=42)\end{array}$ \\
\hline Age & $34.3 \pm 4.4$ & $34.3 \pm 3.7$ & $35.7 \pm 6.8$ & $34.7 \pm 6.8$ & $34.9 \pm 5.0$ \\
\hline BMI & $21.6 \pm 3.7$ & $20.9 \pm 3.4$ & $21.5 \pm 2.7$ & $21.4 \pm 2.9$ & $21.3 \pm 2.9$ \\
\hline Family history (\%) & 93.3 & 81.5 & 57.1 & $56.3^{*}$ & $65.2^{\ddagger}$ \\
\hline Activity score & $1.9 \pm 0.5$ & $2.2 \pm 0.5$ & $2.0 \pm 0.5$ & $1.8 \pm 0.4$ & $2.0 \pm 0.5$ \\
\hline Outdoor score & $11.0 \pm 0.9$ & $10.8 \pm 1.4$ & $10.7 \pm 1.9$ & $10.9 \pm 0.4$ & $10.8 \pm 2.2$ \\
\hline Dietary calcium $(\mathrm{mg} / \mathrm{d})$ & $834 \pm 530$ & $1,158 \pm 458$ & $900 \pm 48$ & $993 \pm 458$ & $1,020 \pm 444$ \\
\hline Dietary vitamin $\mathrm{D}(\mathrm{mg} / \mathrm{d})$ & $177 \pm 172$ & $246 \pm 167$ & $144 \pm 127$ & $179 \pm 67$ & $194 \pm 133$ \\
\hline Caloric intake (kcal/d) & $1,705 \pm 258$ & $1,705 \pm 379$ & $1,736 \pm 472$ & $1,702 \pm 328$ & $1,713 \pm 376$ \\
\hline Serum $25(\mathrm{OH}) \mathrm{D}(\mathrm{ng} / \mathrm{ml})$ & $28.6 \pm 8.9$ & $28.6 \pm 9.5$ & $24.5 \pm 10.7$ & $30.4 \pm 8.7$ & $49.5 \pm 15.6$ \\
\hline Alkaline phosphatase (IU/liter) & $54.6 \pm 16.7$ & $51.8 \pm 18.2$ & $47.2 \pm 17.2$ & $49.3 \pm 12.3$ & $27.8 \pm 9.7$ \\
\hline Serum calcium (mg/dl) & $9.0 \pm 0.34$ & $9.4 \pm 0.5$ & $9.1 \pm 0.1$ & $9.4 \pm 0.4$ & $9.2 \pm 0.4$ \\
\hline Urine calcium $(\mathrm{mg} / 24 \mathrm{~h})$ & $118 \pm 55$ & $167 \pm 95$ & $157 \pm 743$ & $111 \pm 63$ & $147 \pm 81$ \\
\hline
\end{tabular}

${ }^{*} P=0.055 .{ }^{\ddagger} P=0.03$ vs. quartile 1 , respectively (Tukey’s multiple-range test at $95 \%$ confidence limit).

menorrhea and amenorrhea also represent states of transient hypoestrogenism, which, if protracted long enough, might either interfere with normal skeletal maturation or produce subtle bone loss that may not be reversible. In a previous study, Prior et al. (25) observed that anovulatory cycles and defects in the luteal phase could exist even in the presence of normal cycle length and that these asymptomatic disturbances may be associated with significant bone loss, thus suggesting that not only estrogen but progesterone levels may be important for the maintenance of a normal skeletal homeostasis. These observations further underscore the importance of intact ovarian function for an adequate premenopausal bone mass. On the other hand, although hormonal replacement therapy in early postmenopausal women has been documented to prevent rapid bone loss (3), whether the resumption of normal menses in women with temporary but substantially long oligomenorrhea/amenorrhea is sufficient to counterbalance the negative effect of transient hypoestrogenism within the fertile period remains to be determined $(26,27)$.

Our cross-sectional analysis also revealed an inverse relationship between VBD and age at menarche, an observation also reported by others $(13,28)$. In our population, women with normal bone density had their first menstrual period an average 14 mo earlier than women with low bone density.
Thus, achieving sexual maturity earlier in life appears to be an additional factor conditioning the exposure of the skeletal tissue to the beneficial effects of estrogen.

Integrating these two physiological events (i.e., characteristics of menstrual cycles and age at menarche), along with the use of birth control pills, into an ES further evidenced the weight of estrogen exposure on premenopausal bone mass. ES was highly correlated with VBD and, in the multiple-regression analysis, it was the most important independent contributor to VBD variability. Similar results were obtained in younger adolescent females in whom wrist and spine bone densities positively correlated with estrogen status, estimated by a score similar to ours (13). In that study the use of birth control pills was given a heavier weight in the estimate of ES than in our study. However, although a few reports have established a positive relationship between bone density and the use of oral contraceptives $(15,17)$, in another study such a correlation was not evident (16). Besides the different doses of active compounds present in different birth control preparations, one possible explanation for these inconsistent results might stem from the fact that exogenous estrogen may suppress endogenous production, so that circulating levels of active hormones may not be excessively high (29). In addition, orally administered estrogen undergoes rapid hepatic degradation and loss of activity (30),

Table VI. Estrogen Status of Patients Stratified by Quartiles

\begin{tabular}{lccccc}
\hline & Quartile 1 & Quartile 2 & Quartile 3 & Quartile 4 & Quartiles 2-4 \\
\hline ES & $15.3 \pm 4.5$ & $17.7 \pm 3.5$ & $17.9 \pm 2.9$ & $18.9 \pm 1.7^{*}$ & $18.1 \pm 2.7^{\ddagger}$ \\
Age at menarche (yr) & $13.9 \pm 1.9$ & $12.8 \pm 1.5$ & $12.6 \pm 1.2$ & $13.0 \pm 1.6$ & $12.8 \pm 1.4^{\S}$ \\
Menstrual irregularities (\%) & 37.5 & 25.0 & 50.0 & 15.3 & 23.9 \\
BCP (yr of use) & $4.1 \pm 3.8$ & $3.9 \pm 3.8$ & $3.5 \pm 3.1$ & $3.7 \pm 3.7$ & $3.7 \pm 3.4$ \\
Serum estradiol $(\mathrm{pg} / \mathrm{ml})$ & $39.9 \pm 45$ & $62.3 \pm 52$ & $93.8 \pm 51$ & $165.3 \pm 108$ & $219.8 \pm 75$ \\
Serum estrone $(\mathrm{pg} / \mathrm{ml})$ & $104.5 \pm 71$ & $139.8 \pm 57$ & $4.1 \pm 8.4$ & $4.9 \pm 9.0$ & $166.1 \pm 78$ \\
Number of pregnancies & $1.53 \pm 1.1$ & $0.94 \pm 1.2$ & $1.79 \pm 1.7$ & $1.0 \pm 1.1$ & $1.2 \pm 1.4$ \\
Lactation $(\mathrm{yr})$ & $4.3 \pm 8.3$ & $0.94 \pm 1.2$ & & $3.2 \pm 7.2$
\end{tabular}

${ }^{*} P=<0.05 .{ }^{\ddagger} P=0.006 .{ }^{\S} P=0.02$ vs. quartile 1 , respectively (Tukey’s multiple-range test at $95 \%$ confidence limit). 


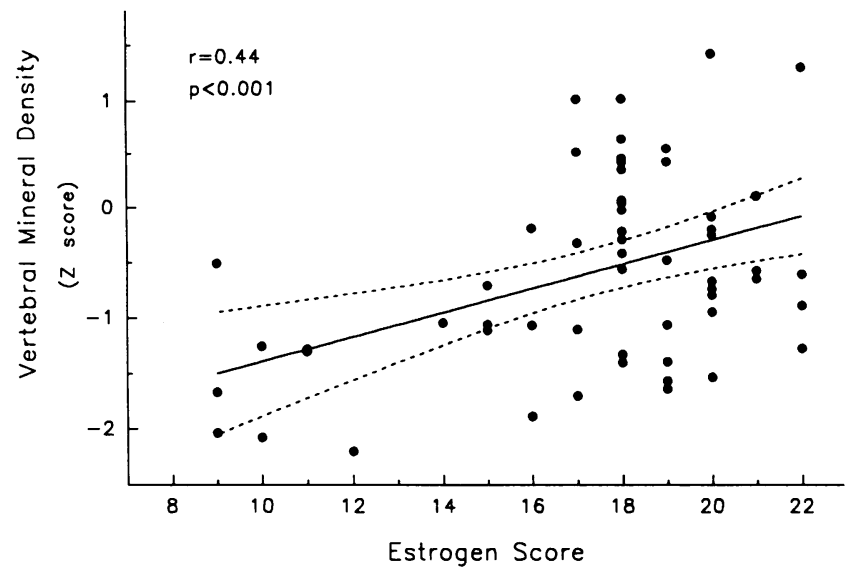

Figure 2. Correlation between vertebral bone density, expressed as $Z$ score, and estrogen score in 57 premenopausal Caucasian women. The regression line and $95 \%$ confidence interval are also illustrated.

which adds another confounding factor. Thus, although the use of oral contraceptives is certainly relevant to the buildup of bone mass in young women, the real long-term effects are not clear at present.

Several reports have shown positive correlations between bone mass and serum estrogen levels (10-12). Our data are consistent with this notion, but they are limited by the fact that hormone levels were obtained randomly during the menstrual cycle, thus they may not be representative of the overall ovarian function of each subject. On the other hand, Dhuper et al. (13) did not find a significant correlation between bone mass and serum estrogen levels measured in a pooled sample of blood obtained at four different days during the cycle. In any case, because of the intrinsic wide variability in the different phases of the menstrual cycle, a single random serum estrogen level has obvious limited value for any diagnostic use for early detection of low bone mass in premenopausal women.

The present study also underscores the role of heredity on bone mass development. Premenopausal women with low bone mass had a positive maternal family history of osteoporosis more frequently than subjects with normal bone mass. This

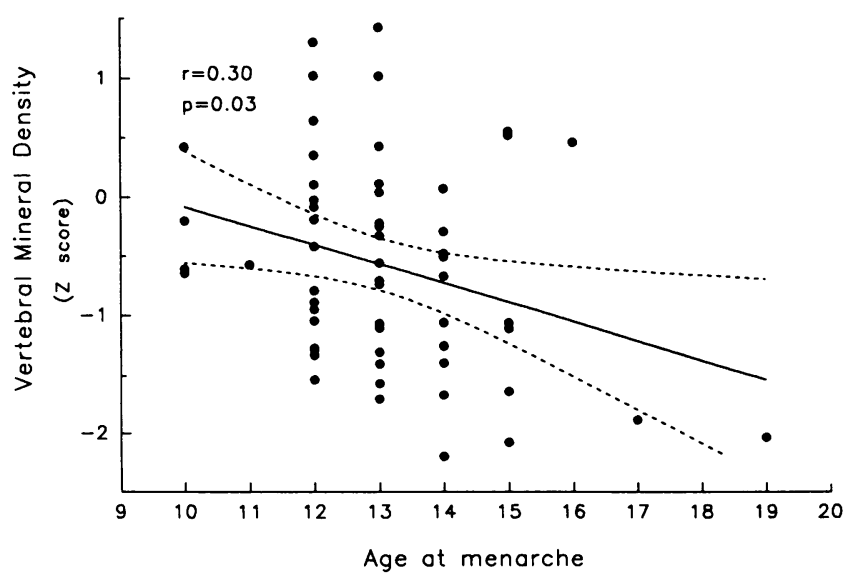

Figure 3. Correlation between vertebral bone density, expressed as $Z$ score, and age at menarche in 58 premenopausal Caucasian women. The regression line and $95 \%$ confidence interval are also illustrated.
Table VII. Correlation Analysis between Vertebral Bone Density and Clinical, Demographic, and Dietary Features of 39 Premenopausal Women

\begin{tabular}{lcc}
\hline \multicolumn{1}{c}{ Variable } & $r$ & Pearson partial $r$ \\
\hline BMI & -0.06 & -0.08 \\
ES & $0.49^{*}$ & $0.57^{*}$ \\
Number of pregnancies & -0.02 & -0.08 \\
Parity & -0.04 & -0.18 \\
Lactation & -0.01 & 0.07 \\
Physical activity & 0.01 & 0.06 \\
Sunlight exposure & -0.13 & -0.22 \\
Dietary calcium & 0.14 & $0.37^{\ddagger}$ \\
Dietary vitamin D & -0.06 & $-0.31^{\S}$ \\
Serum 25(OH)D & 0.07 & 0.07 \\
& & \\
\hline
\end{tabular}

$* P<0.01$

$\ddagger P<0.02$.

${ }^{\S} P<0.05$.

finding is in keeping with previous studies, demonstrating a good correlation in bone densities between mother and daughter pairs $(6,31)$. However, the genetic potential to acquire a certain level of bone mass might not be contributed entirely by the mother's side. A recent study by Matkovic et al. (32) showed a good correlation in bone densities, not only between mothers and daughters but also between fathers and daughters, pointing to a potential contribution of the paternal side to a woman's genetic potential for bone mass development. This possibility has not been thoroughly explored in the past because of the notion that postmenopausal osteoporosis is a condition strictly dependent on female sex hormones, but obviously should be considered in the future.

Of the environmental factors considered in the present study, only dietary calcium intake and, to a lesser extent, vitamin D intake exhibited significant correlations with bone density when correcting for the other factors. Although the beneficial effect of an increased calcium intake has been reported in short-term prospective studies (33), the negative correlation between dietary vitamin D and VBD remains difficult to interpret. Nevertheless, neither dietary habits nor any of the other environmental factors, i.e., body mass, physical activity, and sunlight exposure, provided a significant source of VBD variance in the multiple-correlation analysis, indicating that these

Table VIII. Stepwise Selection Analysis of Variables Contributing to Vertebral Bone Density in 39 Premenopausal Women: Final Model

\begin{tabular}{ccccc}
\hline $\begin{array}{c}\text { Independent } \\
\text { variable }\end{array}$ & Coefficient & SE & \multicolumn{1}{c}{$t$} & $P$ \\
\hline Constant & -3.013 & 0.683 & -4.42 & 0.0001 \\
ES & 0.128 & 0.037 & 3.43 & 0.0015
\end{tabular}

$R^{2}=0.24$; adjusted $R^{2}=0.22$.

Standard error of estimates $=0.749$; mean absolute error $=0.626$. Variables considered but not included in the model: BMI, number of pregnancies, parity, lactation, physical activity, sunlight exposure, dietary calcium, dietary vitamin $\mathrm{D}$, serum $25(\mathrm{OH}) \mathrm{D}$. 
factors have little predictive value for premenopausal bone density. However, cross-sectional studies are limited by the fact that data acquired at a single point in time may not necessarily reflect lifelong behaviors. In fact, a positive effect of physical activity and weight bearing on bone mass have been reported $(34,35)$. Likewise, although we did not find a strong contribution of body mass to bone density, heavier subjects have been reported to have denser bones than individuals with lower body mass $(13,36)$. However, the effect of body size on skeletal maturation and health may also be mediated by hormonal, as well as mechanical effects. Overweight individuals tend in fact to have higher estrogen levels (36), which may add to the positive effect of weight bearing on bone density.

Finally, our data do not reveal significant age-related changes in bone density between 20 and $40 \mathrm{yr}$ of age. This is in agreement with previous studies $(28,37)$ and may simply reflect the lack of power of cross-sectional observations to detect subtle historical trends, such as those leading to a premenopausal peak of bone mass. Alternatively, the results may indicate that between 20 and $40 \mathrm{yr}$ of age there is no significant increase of bone density and that a peak is reached earlier. The answer to this question can be obtained only through longterm longitudinal analyses.

The notion that in some otherwise healthy women bone density is lower than average well before menopause occurs implies that a certain number of cases diagnosed as postmenopausal osteoporosis may in fact be cases of women whose bone density was low before menopause and came to medical observation only after the cessation of menses, perhaps because of a vertebral fracture. Thus, a low bone mass in an early postmenopausal woman should not necessarily be interpreted to indicate rapid bone loss. Careful evaluation of a patient's past history with respect to menstrual history and familiarity of osteoporosis is very helpful and should always be considered a major point in the diagnostic process of postmenopausal women with low bone mass. If these assumptions are confirmed in larger, prospective studies, revision of the current etiologic classification of osteoporosis should be considered to indicate that type 1 (or postmenopausal) osteoporosis may be the consequence of either fast bone loss or low peak bone mass.

In conclusion, the present study demonstrates that estrogen status is the most important determinant of premenopausal bone mass. Other factors, such as body mass, dietary habits, physical activity, and sunlight exposure, appear to have less impact. A thorough assessment of menstrual history may therefore help in the evaluation of bone health in premenopausal women. Thus, young Caucasian females with a positive family history of osteoporosis and with signs and symptoms suggestive of hormonal imbalance should be considered for screening for osteopenia and advised for possible intervention after menopause. Efforts directed toward the preservation of bone mass and the prevention of bone loss should be initiated as early as possible.

\section{Acknowledgments}

The authors wish to thank Jill Stoll, RD, for the dietary analyses; Reta Rupich, $\mathrm{PhD}$, and Michael Griffin, $\mathrm{PhD}$, for the bone density measurements; and JoAnn Pierson for secretarial assistance.

\section{References}

1. Chesnut, C. H. 1991. Theoretical overview: bone development, peak bone mass, bone loss, and fracture risk. Am. J. Med. 91 (Suppl. 5B):2S-4S.

2. Gruber, H. E., J. L. Ivey, D. J. Baylink, M. Matthews, W. B. Nelp, K. Sisom, and C. H. Chesnut III. 1984. Long-term calcitonin therapy in postmenopausal osteoporosis. Metab. Clin. Exp. 33:295-303.

3. Lindsay, R., J. M. Aitken, J. B. Anderson, D. M. Hart, E. B. MacDonald, A. C. Clarke. 1976. Long-term prevention of postmenopausal osteoporosis by oestrogen. Lancet. i:1038-1040.

4. Watts, N. B., S. T. Harris, H. K. Genant, R. D. Wasnich, P. D. Miller, R. D. Jackson, A. L. Licata, P. Ross, G. C. Woodson, M. J. Yanover, et al. 1990. Intermittent cyclical etidronate treatment of postmenopausal osteoporosis. $N$. Engl. J. Med. 323:73-79.

5. Ott, S. M. 1990. Editorial. Attainment of peak bone mass. J. Clin. Endocrinol. \& Metab. 71:1082A-1082C.

6. Seeman, E., J. L. Hooper, L. A. Bach, M. E. Cooper, E. Parkinson, J. McKay, G. Jerums. 1989. Reduced bone mass in daughters of women with osteoporosis. N. Engl. J. Med. 320:554-558.

7. Smith, D. M., W. E. Nance, K. W. Kang, J. C. Christian, C. C. Johnston. 1973. Genetic factors in determining bone mass. J. Clin. Invest. 52:2800-2808.

8. Pocock, N., J. A. Eisman, J. L. Hopper, M. G. Yeates, P. N. Sambrook, S. Eberi. 1987. Genetic determinants of bone mass in adults. J. Clin. Invest. 80:706710 .

9. Lindsay, R. 1991. Estrogens, bone mass, and osteoporotic fracture. Am. J. Med. 91 (Suppl. 5B):10S-13S.

10. Steinberg, K. K., L. W. Freni-Titulaer, E. G. Depuey, D. T. Miller, D. S. Sgoutas, C. H. Coralli, D. L. Phillips, T. N. Rogers, R. V. Clark. 1989. Sex steroids and bone density in premenopausal and perimenopausal women. J. Clin. Endocrinol. \& Metab. 69:533-539.

11. Buchanan, J. B., C. Myers, T. Lloyd, P. Leuenberger, L. M. Demers. 1988. Determinants of peak trabecular bone density in women: the role of androgens, estrogen, and exercise. J. Bone Miner. Res. 3:673-680.

12. Sowers, M. F. R., B. Shapiro, M. A. Gilbraith, M. Jannausch. 1990. Health and hormonal characteristics of premenopausal women with lower bone mass. Calcif. Tissue Int. 47:130-135.

13. Dhuper, S., M. P. Warren, W. J. Brooks-Gunn, R. Fox. 1990. Effects of hormonal status on bone density in adolescent girls. J. Clin. Endocrinol. \& Metab. 71:1083-1088.

14. Hreshchyshyn, M. M., A. Hopkins, S. Zylstra, M. Anbar. 1988. Associations of parity, breast-feeding, and birth control pills with lumbar spine and femoral neck bone densities. Am. J. Obstet. Gynecol. 159:318-322.

15. Lindsay, R., J. Tohme, B. Kanders. 1986. The effect of oral contraceptive use on vertebral bone mass in pre- and post-menopausal women. Contraception. 34:333-339.

16. Lloyd, T., J. R. Buchanan, G. R. Ursino, C. Myer, G. Woodward, D. R. Halbert. 1989. Long-term oral contraceptive use does not affect trabecular bone density. Am. J. Obstet. Gynecol. 160:402-404.

17. Goldsmith, N. F., and J. O. Johnston. 1975. Bone mineral: effects of oral contraceptives, pregnancy and lactation. J. Bone Jt. Surg. Am. Vol. 57A:657658.

18. Villareal, D. T., R. Civitelli, A. Chines, L. V. Avioli. 1991. Subclinical vitamin $\mathrm{D}$ deficiency in postmenopausal women with low vertebral bone mass. $J$. Clin. Endocrinol. \& Metab. 72:628-634.

19. Block, J. E., R. Smith, C. C. Glueer, P. Steiger, B. Ettinger, H. K. Genant. 1989. Models of spinal trabecular bone loss as determined by quantitative computed tomography. J. Bone Miner. Res. 4:249-257.

20. Pacifici, R., R. C. Rupich, L. V. Avioli. 1990. Vertebral cortical bone mass measurement by a new quantitative computer tomography method: correlations with vertebral trabecular bone measurements. Calcif. Tissue Int. 47:215-220.

21. Haddad, J. G., K. J. Chyu. 1971. Competetive protein-binding radioassay for 25-hydroxycholecalciferol. J. Clin. Endocrinol. \& Metab. 33:992-995.

22. Cann, C. E., D. J. Cavanaugh, K. Scnurpfiel, M. C. Martin. 1988. Menstrual history is the primary determinant of trabecular bone density in women. Med. Sci. Sports Exercise. 20(Suppl. 2):S59. (Abstr.)

23. Cann, C. E., M. C. Martin, H. K. Genant, R. B. Jaffe. 1984. Decreased spinal mineral content in amenorrheic women. JAMA (J. Am. Med. Assoc.). 251:626-629.

24. Drinkwater, B. L., B. Bruemner, C. H. Chesnut. 1990. Menstrual history as a determinant of current bone density in young athletes. JAMA (J. Am. Med. Assoc.). 263:545-548.

25. Prior, J. C., Y. M. Vigna, M. T. Schecter, A. E. Burgess. 1990. Spinal bone loss and ovulatory disturbances. N. Engl. J. Med. 323:1221-1227.

26. Drinkwater, B. L., K. Nilson, S. Ott, C. H. Chesnut III. 1986. Bone mineral density after resumption of menses in amenorrheic women. JAMA (J. Am. Med. Assoc.). 256:380-382.

27. Bachrach, L. K., D. K. Katzman, I. F. Litt, D. Guido, R. Marcus. 1991. 
Recovery from osteopenia in adolescent girls with anorexia nervosa. J. Clin. Endocrinol. \& Metab. 72:602-606.

28. Rosenthal, D. I., W. Mayo-Smith, C. W. Hayes, J. S. Khurana, B. M. K. Biller, R. M. Neer, A. Klibanski. 1989. Age and bone mass in premenopausal women. J. Bone Miner. Res. 4:533-538.

29. Mishell, D. R., I. H. Thorneycroft, R. M. Nakamura, Y. Nagata, S. Stone. 1972. Serum estradiol in women ingesting combination oral contraceptive steroids. Am. J. Obstet. Gynecol. 114:923-928.

30. Humpel, M., B. Nieuweboer, H. Wendt, U. Speck. 1979. Investigations of pharmacokinetics of ethinylestradiol to specific consideration of a possible firstpass effect in women. Contraception. 19:421-431.

31. Tylavsky, F. A., A. D. Bortz, R. L. Hancock, J. J. B. Anderson. 1989 Familial resemblance of radial bone mass between premenopausal mothers and their college-age daughters. Calcif. Tissue Int. 45:265-272.

32. Matkovic, V., D. Fontana, C. Tominac, P. Goel, C. Chesnut. 1990. Factors that influence peak bone mass formation: a study of calcium balance and the inheritance of bone mass in adolescent females. Am. J. Clin. Nutr. 52:878-888.
33. Baran, D., A. Sorensen, J. Grimes, R. Lew, A. Karellas, B. Johnson, J. Roche. 1990. Dietary modification with dairy products for preventing vertebral bone loss in premenopausal women: a three-year prospective study. J. Clin. Endocrinol. \& Metab. 70:264-270.

34. Davee, A. M., C. J. Rosen, R. A. Adler. 1990. Exercise patterns and trabecular bone density in college women. J. Bone Miner. Res. 5:245-250.

35. Dalsky, G. P., K. S. Stocke, A. A. Ehsani, E. Slatopolsky, W. C. Lee, S. J. Birge. 1988. Weight-bearing exercise training and lumbar bone mineral content in postmenopausal women. Ann. Intern. Med. 108:824-828.

36. Liel, Y., J. Edwards, J. Shary, K. M. Spicer, L. Gordon, N. H. Bell. 1988. The effects of race and body habitus on bone mineral density of the radius, hip, and spine in premenopausal women. J. Clin. Endocrinol. \& Metab. 66:12471250.

37. Mazess, R. B., and H. S. Barden. 1991. Bone density in premenopausa women: effects of age, dietary intake, physical activity, smoking, and birth-control pills. Am. J. Clin. Nutr. 53:132-142. 